\title{
Phlebotomus perfiliewi Parrot, 1930 (Diptera, Psychodidae) dans le sud-est de la France
}

\author{
IZRI M.A.*, MARTY P.**, FAURAN P. ***, LE FICHOUX Y.** et ROUSSET J.-J.*
}

Summary : PHLEBOTOMUS PERFILIEWI PARROT, 1930 (DIPTERA, PSYCHODIDAE) IN THE SOUTH-EAST OF France

The authors report the result of an entomological study in the southeastern France. It is the first time that Phlebotomus perfiliewi is described in that country.

KEY WORDS : Phlebotomus perfiliewi. France.

ix espèces de Phlébotomes étaient connues jusqu'à ce jour en France continentale (Rioux et al., 1967; Rioux et al., 1982) : Phlebotomus papatasi Scopoli, 1786, P. ariasi Tonnoir, 1921, P. perniciosus Newstead, 1911; P. mascittii Grassi, 1908, P. sergenti Parrot, 1917 et Sergentomyia minuta Rondani, 1843. Nous ajoutons aujourd'hui une septième espèce: Phlebotomus perfiliewi Parrot, 1930.

Du ler au 31 Juillet 1993, nous avons effectué une enquête entomologique sur les Phlébotomes de la Principauté de Monaco et de quatre communes françaises voisines. Deux-cent-deux nuits/pièges CDC et quatre $\mathrm{m}^{2}$ de pièges adhésifs ont permis la capture de, respectivement, 1279 et 210 Phlébotomes.

Cinq espèces sont identifiées (Tableau ci-dessous).

Phlebotomus perfiliewi ơa été capturé à l'aide d'un piège CDC sur la terrasse d'une habitation semi-rurale de la commune de Beausoleil à moins de 100 mètres de la frontière monégasque (altitude : $60 \mathrm{~m}$., exposition sud).

Signalée pour la première fois en France, cette espèce est répandue en Italie (Biocca et al., 1972) où son rôle vecteur y est reconnu (Maroli et al., 1987). Rare au Maroc, elle est en revanche fréquente en Tunisie et en Algérie où elle a été trouvée naturellement infestée par Leishmania infantum MON-24 (Izri et Belazzoug, 1993).

\section{Résumé}

les auteurs rapportent les résultats d'une enquête entomologique dans le sud-est de la France. Phlebotomus perfiliewi est signalé pour la première fois dans ce pays.

MOTS CLES : Phlebotomus perfiliewi, France

L'unique exemplaire trouvé à Beausoleil ne permet pas de statuer sur le rôle épidémiologique de cette espèce. Etant donné son absence dans le reste du midi méditerranéen, il s'agit probablement d'une station ectopique. Sa présence à Beausoleil rend nécessaires d'autres recherches sur le même site et dans la région.

\section{RÉFÉRENCES}

Biocca E., Coluzzi A. et Constantini R. : Distribution des différentes espèces de phlébotomes en Italie et transmission des leishmanioses et de quelques arboviroses. Colloques internationaux du CNRS, 1972, n² 239, 157-166.

IzRi M.A. et Belazzoug S. : Phlebotomus (Larroussius) perfiliewi naturally infected with dermotropic Leishmania infantum in Tenes, Algeria. Transactions of the Royal Society of Tropical Medicine and Hygiene, 1993, 87, 399.

Maroli M., Gramiccia M. et Gradoni L. : Natural infection of Phlebotomus perfiliewi with Leishmania infantum in a cutaneous leishmaniasis focus of the Abruzzi region, Italy. Transactions of the Royal Society of Tropical Medicine and Hygiene, 1987, 81, 596-598.

Rioux J.A. et al. : Ecologie des leishmanioses dans le sud de la France. 1. - Les phlébotomes. Annales de Parasitologie Humaine et Comparée, 1967, 42, n 6, 561-603.

Rioux J.A., Jarry D., MaAzoun R. et Wallbanks K. : Confirmation de l'existence en France de Phlebotomus sergenti Parrot, 1917. Annales de Parasitologie Humaine et Comparée, 1982, 57, 647-648.

Accepté le 15 juin 1994

Répartition des captures selon le type de piège utilisé, l'espèce et le sexe des Phlébotomes

\begin{tabular}{|c|c|c|c|c|c|}
\hline \multirow{2}{*}{ espèce } & \multicolumn{2}{|c|}{ pièges CDC } & \multicolumn{2}{|c|}{ pièges adhésifs } & \multirow{2}{*}{ Total } \\
\hline & mâles & femelles & mâles & femelles & \\
\hline P. perniciosus & 657 & 381 & 16 & 4 & 1058 \\
\hline P. ariasi & 23 & 17 & & & 40 \\
\hline P. mascittii & & 1 & & & 1 \\
\hline$P$. perfiliewi & 1 & & & & 1 \\
\hline S. minuta & 79 & 120 & 109 & 81 & 389 \\
\hline Total & 760 & 519 & 125 & 85 & 1489 \\
\hline
\end{tabular}

\footnotetext{
" Parasitologie-Mycologie, Hôpital Avicenne, 93009 BOBIGNY cedex.

** Parasitologie-Mycologie, Faculte de Medecine, 06107 NICE cedex 2.
} 